\title{
Tratamiento del priapismo recurrente de bajo flujo mediante autoinyección intracavernosa domiciliaria de metoxamina
}

\author{
J. Rodríguez Tolrà, J.Ma Cuadrado Campaña, Ll. Fumadó Ciutat, E. Franco Miranda \\ Unidad de Andrología. Servicio de Urología. Hospital Universitario de Bellvitge. Hospitalet de Llobregat. Barcelona.
}

Actas Urol Esp 2006; 30 (3): 324-325

\section{RESUMEN}

TRATAMIENTO DEL PRIAPISMO RECURRENTE DE BAJO FLUJO MEDIANTE AUTOINYECCIÓN INTRACAVERNOSA DOMICILIARIA DE METOXAMINA

Presentamos un caso de priapismo recurrente de bajo flujo de etiología desconocida; comprobado durante su ingreso hospitalario la eficacia y buena tolerancia a la administración intracavernosa de metoxamina, se aleccionó al paciente en la autoinyección domiciliaria, realizando ésta durante aproximadamente un mes, con desaparición del priapismo recurrente. El paciente conservó su función eréctil.

Palabras clave: Priapismo recurrente. Autoinyección domiciliaria. Metoxamina.

\section{ABSTRACT}

INTRACAVERNOSAL METHOXAMINE SELF-INJECTION FOR THE TREATMENT OF LOW-FLOW RECURRENT PRIAPISM

We report the case of a young man with a recurrent idiopathic priapism, successfully treated with methoxamine intracarvenosal self-injections. The patient was instructed in intracorporeal self-injection of this pure alpha-1 adrenergic agonist, which provided complete detumescence. The patient became asymptomatic after one month of domiciliary treatment. We conclude that intracavernosal self-administered methoxamine can be a useful treatment of recurrent idiopathic priapism.

Keywords: Recurrent idiopathic priapism. Self-injection. Methoxamine.

$\mathrm{E}$ 1 priapismo recurrente de bajo flujo es aquella erección espontánea y prolongada, con una duración de hasta 3 horas y que suele acabar con detumescencia también espontánea del pene.

Tiene un alto riesgo de desarrollar priapismo de bajo flujo, sobre todo si el paciente está en tratamiento con psicofármacos o antihipertensivos.

Se trata generalmente de pacientes con bajo deseo sexual a los cuales su situación les angustia y piden solución a su problema.

La fisiopatología de esta enfermedad no es bien conocida, pero se sabe que responde a los agonistas alfa adrenérgicos, y serán ellos los que nos ayudarán a solucionar el problema.

\section{CASO CLÍNICO}

Paciente de 34 años que ingresó para estudio de un priapismo recurrente de bajo flujo, sin antecedentes patológicos de interés. No fue posible demostrar una etiología asociada a su enfermedad.

Debido a la buena respuesta a la administración intracavernosa de entre 2-10 mg de metoxamina, con excelente tolerancia pero con recurrencia del priapismo, se aleccionó al paciente en la autoinyección domiciliaria con dicho fármaco.

Fue necesaria su utilización varias veces durante aproximadamente un mes; posteriormente el paciente ha conservado sin problemas la función eréctil.

\section{DISCUSIÓN}

Los fármacos ideales para revertir una erección prolongada son los agonistas adrenérgicos, no obstante deberemos distinguir entre los mixtos, que también tienen actividad beta y que por tanto serían responsables de efectos adversos sistémicos 
tales como taquicardia, arritmias, angor, edema pulmonar etc. ${ }^{1}$, de aquellos agonistas alfa adrenérgicos puros que por no tener actividad beta no van ha producir efectos adversos, siendo por tanto los idóneos para el tratamiento domiciliario en caso de un priapismo recurrente de bajo flujo.

En la Tabla 1 recogemos los agonistas adrenérgicos utilizados mas habitualmente, indicando su actividad alfa y beta y las dosis aconsejables de uso.

El priapismo recurrente de bajo flujo es una entidad muy poco frecuente y su tratamiento domiciliario es casi obligado debido precisamente a su recurrencia. Se han venido utilizando por diversos autores algunos de los fármacos enumerados en la Tabla 1, pero siempre las publicaciones tratan de casos aislados. Así Van Driel $^{2}$ y Steingerg $^{3}$ presentan cada uno un caso que se solucionó con Epinefrina, y Mc Donald ${ }^{4}$ un caso exitoso con Metaraminol, no obstante no se puede olvidar que precisamente con este último fármaco, utilizado en casos de priapismo se han descrito muertes como consecuencia de roturas de aneurismas, atribuibles a su actividad ${ }^{5}$. Por tanto creemos que es preferible la utilización de agonistas alfa puros; dentro de estos un fármaco que reuniría las condiciones ideales sería la Fenilefrina, ya que además su mecanismo de acción es corto, Dittrich $^{6}$ la ha utilizado con éxito en varios casos. También aparece un caso publicado por Ralph ${ }^{7}$ que utilizó la fenilefrina mediante una bomba de autoinyección situada en el escroto y conectada a una cánula insertada en el cuerpo cavernoso, el paciente lo utilizó durante cuatro meses.

Desgraciadamente en nuestro país la Fenilefrina sólo podemos conseguirla como medicamento extranjero, por lo que nosotros utilizamos con buenos resultados la Metoxamina, de características muy parecidas a la Fenilefrina, aunque con una

Tabla 1

\begin{tabular}{|c|c|c|c|c|}
\hline Fármaco & $\begin{array}{c}\text { Activi } \\
\text { Alfa }\end{array}$ & $\begin{array}{l}\text { dad adr } \\
\text { Betal }\end{array}$ & $\begin{array}{l}\text { enérgica } \\
\text { Beta2 }\end{array}$ & Dosis \\
\hline Epinefrina & +++ & +++ & +++ & $10-20 \mathrm{mcg}$ \\
\hline Norepinefrina & +++ & ++ & ++ & $10-20 \mathrm{mcg}$ \\
\hline Efedrina & + & ++ & ++ & $50-100 \mathrm{mcg}$ \\
\hline Metaraminol & ++ & + & + & $2-4 \mathrm{mlg}$ \\
\hline Etilefrina & ++ & + & + & $5-10 \mathrm{mlg}$ \\
\hline Fenilefrina & +++ & 0 & & $100-500 \mathrm{mcg}$ \\
\hline Metoxamina & +++ & 0 & 0 & $2-10 \mathrm{mlg}$ \\
\hline
\end{tabular}

vida media un poco mas larga, por tanto creemos que en nuestro medio sería precisamente este fármaco el de elección para tratar domiciliariamente un priapismo recurrente de bajo flujo.

También se han utilizado otros fármacos para el tratamiento de éste tipo de priapismo como son la Gabapentina ${ }^{8}$ y el Baclofeno ${ }^{9}$ por vía oral, administrados en periodos prolongados, seis meses o más.

Finalmente concluiremos diciendo que en caso de querer establecer un tratamiento domiciliario mediante inyecciones intacavernosas de metoxamina o cualquier otro agonista alfa adrenérgico, sería necesario ingresar al paciente inicialmente para comprobar primero la eficacia de la medicación y la falta de efectos secundarios, en segundo lugar, ajustar la dosis mínima necesaria para revertir el episodio de priapismo cuando se presente, y aleccionar al enfermo sobre la forma adecuada de autoinyectarse. Una vez dado de alta deberemos realizar un seguimiento periódico hasta la total remisión de la enfermedad.

\section{REFERENCIAS}

1. Mary Lee, Bradley Cannon, Roohollah Sharifi. Chart for preparation of dilutions of alfa-adrenergic agonists for intracavernous use in treatment of priapism. J Urol1995;153:1182-1183.

2. Van Driel MF, Joosten EA, Mensink HJ. Intracorporeal self-injection with epinephrine as treatment for idiopathic recurrent priapism. Eur Urol 1990;17:95-97.

3. Steinberg J, Eyre RC. Management of recurrent priapism with epinephrine sel-injection and gonadotropin releasing hormone analoghe. J Urol 1995;153:152-153.

4. McDonald M, Santucci RA. Manejo exitoso del priapismo de bajo flujo recurrente mediante autoinyección domiciliaria del alfa-agonista metaraminol. Rev Urol 2004;5(1):49-50

5. Watters GR, Keogh EJ, Carati CJ, Earle CM, Wisniewski ZS, Tulloch AG, et al. Prolonged erections following intracorporeal injections of medications to overcome impotence. Br. J Urol 1988;62:173-175.

6. Diettrich A, Albrecht K, Bar-Moshe O, Vandendris M. Treatment of pharmacological priapism with phenylephrine. J Urol 1991;146:323-324.

7. Ralph DJ, Pescatori ES, Brindley GS, Prior JP. Intracavernosal phenylephrine for recurrent priapism: self-administration by drug delivery implant. J Urol 2001;165:1632.

8. Perimenis P, Athanasopoulos A, Papathanasopoulos P, Barbalias G. Gabapentin in the management of the recurrent, refractory, idiopatic priapism. Int J Impot Res 2004;16(1):84-85.

9. Vaidyanathan S, Watt JW, Singh G, Hughes PL, Selmi F, Oo T, et al. Management of recurrent priapism in a cervical spinal cord injury patient with oral baclofen therapy. Spinal Cord 2004;42(2):134-135.

Dr. R. Rodríguez Tolrà

Hospital Universitario de Bellvitge

Feixa Llarga, s/n

08907 Hospitalet de Llobregat (Barcelona)

E-mail: jrtol@wanadoo. es

(Trabajo recibido el 15 de junio 2005) 